\title{
ELEMENTOS CONDICIONANTES DE LA DIVULGACIÓN DE INFORMACIÓN DEL CAPITAL ESTRUCTURAL TECNOLÓGICO1,2
}

\author{
Edila Eudemia Herrera ${ }^{3}$ \\ Clea Beatriz Macgnan ${ }^{4}$
}

\begin{abstract}
Resumen: El estudio analiza los elementos condicionantes de la divulgación de información representativa del capital estructural tecnológico, de los bancos registrados en el mercado de capitales: en Brasil y en España. La divulgación voluntaria de informaciones respecto a este tipo de recursos intangibles pudiera reducir el problema de la asimetría de la información entre el agente y el principal. De una parte se encuentra la teoría de la agencia que presupone que revelar voluntariamente informaciones reduce este problema. De otra, la teoría del coste del propietario presupone que divulgar informaciones produce costes de sistematización y publicación de informaciones, también sumado al hecho de la posible pérdida de ventajas competitivas de la organización, en este caso, los bancos. Este estudio analiza 174 informes anuales, de una muestra de 29 bancos, durante el período del 2006 al 2011. Se utilizó la técnica de regresión lineal múltiple, a través del método de mínimos cuadrados con datos de panel. Se confirman las hipótesis de que el tamaño, la rentabilidad , la antigüedad y la cotización en más de una bolsa son elementos condicionantes de la divulgación de información representativa del capital estructural tecnológico. Lo que quiere decir que los directivos de grandes bancos de Brasil y de España, con mayor rentabilidad, más antigüos, y los que cotizan en más de una bolsa, revelarían voluntariamente informaciones, representativas del capital estructural tecnológico, según el presupuesto de que la reducción de asimetría de información entre ellos y los accionistas minimizarían costes de agencia. Con ello, estarían contrariando el presupuesto de que la revelación de información representaría costes a los propietarios de los bancos.
\end{abstract}

Palabras clave: Capital estructural tecnológico. Recursos intangibles. Asimetría de información, teoría de agencia, teoría del coste del propietario.

\footnotetext{
'Este artigo foi vencedor do prêmio do Melhor Trabalho Apresentado no Spanish Track do VIII Congresso ANPCONT, 2014

${ }^{2} \mathrm{O}$ trabalho é originado de uma pesquisa patrocinada pelo Banco Mundial.

${ }^{3}$ E-mail: eherreradeleon@hotmail.com. Universidad de Panamá

${ }^{4}$ E-mail: cleabeatrizm@gmail.com. Curso de Pós-graduação - Doutorado e Mestrado em Ciências Contábeis da Unisinos

- DOI: http://dx.doi.org/10.14392/asaa.2015080203

- Artigo recebido em 16/09/2014. 2" rodada: 28/11/2014. Aprovado em: 01/08/2015.
} 


\title{
CONDITIONING ELEMENTS OF DISCLOSURE OF THE TECHNOLOGICAL STRUCTURAL CAPITAL
}

\begin{abstract}
The study verifies the conditioning elements of disclosure of information representative of technological structural capital, banks reported in the capital market: in Brazil and Spain. The voluntary disclosure of information regarding this type of intangible resources could reduce the problem of the asymmetry of information between the agent and the principal. A part is the theory of the Agency which presupposes that voluntarily disclose information reduces this problem. Another, the theory of the cost of the owner assumes that spreading information produces costs of systematization and publication of information, also coupled with the fact of the possible loss of competitive advantages of the organization, in this case, the banks. This study analyzes 174 annual reports, a sample of 29 banks, during the period from 2006 to 2011. The technique of multiple linear regressions using the method of least squares with panel data was used. Was confirmed the hypothesis that the size, the profitability, antique and the listing on more than one stock exchange are representative of technological structural capital disclosures conditioning elements. Which means, that managers of large banks of Brazil and Spain, with higher profitability, oldest and which are listed in more than one bag, would voluntarily reveal information, representing the technological structural capital, according to the budget that the reduction of asymmetry of information between them and shareholders minimize costs of Agency. Therefore they would be contrary to the budget that disclosure of information would represent costs to the owners of the banks.
\end{abstract}

Keywords: Technological structural capital; intangible resources; asymmetry of information; agency theory; theory of the cost of the owner 


\section{INTRODUCCIÓN}

-

a severa crisis económica de la década del 2000, que originó una serie de escándalos corporativos, que afectaron la economía mundial. Estos escándalos surgieron en el 2002, y a partir de allí, se han planteado cuestiones profundas sobre las organizaciones y el interés general. (Champlin \& Knoedler, 2003). Esta sucesión de escándalos ha generado preocupación por parte de los reguladores, adquiriendo este tema cada día mayor relevancia. A partir de allí se mostró la necesidad de mayor transparencia en el comportamiento bancario. Tanto es así que ha surgido una nueva propuesta como marco regulatorio de Basilea III, propuesto por el Comité de Supervisión Bancaria de Basilea en 2010; a fin de lograr la medición, normalización y seguimiento del riesgo de liquidez a nivel internacional.

En este ámbito ha surgido el concepto de gobierno corporativo (GC), con la expectativa de aliviar el problema de la agencia, al restringir los incentivos de los directivos para promover sus propios intereses a expensas de los accionistas. (Shleifer \& Vishny, 1997). El GC nació como un mecanismo de equilibrio de poder entre los directivos de las organizaciones y los stakeholders.

El GC emergió con la Ley Sarbanes-Oxley. Esta ley representaría un intento legislativo de gran alcance, para mejorar la calidad de la información financiera en los Estados Unidos. Esto, en respuesta a la cantidad de fraudes contables de finales de 1990 y principios de 2000. (Klein, 2003, Bhagat \& Bolton, 2013). El GC, estaría enfocado en la calidad, la transparencia y la fiabilidad de las relaciones entre los agentes y stakeholders. En base de ello, hablar de cumplimiento de las normas de gobierno corporativo, significaría pensar en que las organizaciones reflejarían más responsabilidad y transparencia con los accionistas. (Adiloğlu \&Vuran, 2012). De esta forma, los accionistas y stakeholders pudieran monitorear el desempeño de las organizaciones, mediante la reducción de la asimetría de la información. (Sanan \& Yodav, 2011). El fin sería que pudiera generarse la tranquilidad necesaria para todos aquellos que tuvieran intereses en el mercado de valores. La aparición de códigos de GC, el cumplimiento obligatorio de las normas contables de mayor la transparencia y el surgimiento de un nuevo orden contable, probablemente no hubiera sido posible, si no hubiese ocurrido la ruina de organizaciones corporativas. (Islam, Cooper \& Andrew, 2006).

En este nuevo orden contable en cuanto a la necesidad de informaciones, se plantearon dos teorías en esta investigación, para analizar las motivaciones de los agentes en cuanto a la revelación de informaciones, siendo estas la teoría de la agencia y la teoría del coste del propietario. La teoría de la agencia presupone que si los intereses entre los accionistas y gerentes no se encuentran alineados, podrían surgir problemas de agencia, es decir que los gerentes busquen satisfacer sus propios intereses a expensas de los intereses de los accionistas. (Jensen; Meckling, 1976). Esto provocaría el surgimiento de costes de agencia. La divulgación voluntaria de información representativa del capital estructural tecnológico busca reducir el problema de la asimetría de información y de los costes de agencia. Por otra parte, la teoría del coste del propietario presupone que divulgar voluntariamente informaciones sobre recursos intangibles genera costes para el propietario, además de que se pudiera estar revelando información estratégica de la empresa, con la consecuente pérdida de sus ventajas competitivas (Verrecchia, 1983; Macagnan, 2007). Esto llevaría a los directivos a reducir el nivel de revelación de información, sea por la posibilidad de pérdida de competencia o por el costes de sistematizar y divulgar información. 
Así, que la pregunta de investigación es: cuáles son los factores explicativos del nivel de revelación de información sobre recursos intangibles representativos de capital estructuural tecnológico en los informes anuales, durante el período 2006 al 2011, de los bancos inscritos en las Bolsas de Valores de Brasil y España. Por tanto el objetivo deste artículo es analizar los elementos condicionantes de la revelación de información sobre recursos intangibles representativos de capital estructuural tecnológico en los informes anuales, durante el período 2006 al 2011, de los bancos inscritos en las Bolsas de Valores de Brasil y España.

La relevancia de este estudio ha sido reconocida por el Banco Mundial que ha financiado la misma, lo que muestra el marcado interés de parte de organismos internacionales de prestigio por el desarrollo de este tipo de investigaciones.

La metodología de este artículo busca analizar cuáles son los elementos condicionantes de la divulgación de información representativa del capital estructural tecnológico, de una muestra compuesta por los bancos inscritos en los mercados de capitales de Brasil (BM\&F-Bovespa), y de España (las bolsas de Madrid y de Barcelona), durante el período 2006-2011.Se utilizó un índice de divulgación, que es un indicador numérico que mide la cantidad de información divulgada por la empresa. Este índice estuvo representado por 20 indicadores representativos del capital estructural tecnológico. Cada indicador toma el valor de uno si el banco divulga información respecto al indicador y cero en caso contrario.

Encontramos que entre mayor sea el tamaño del activo de los bancos, mayor sería la revelación de informaciones sobre sus recursos intangibles representatvos de capital estructural tecnológico. En este sentido, Arvidsson (2003) encontró los mismos resultados en su investigación al analizar empresas de Dinamarca, Finlandia, Noruega y Suecia, pertenecientes a la industria farmacéutica y biotecnología. Por su parte, Rodríguez (2004) concluyó con los mismos resultados al analizar empresas no financieras que cotizan en el mercado continuo español. Estos resultados fueron ratificados por García-Meca et al. (2005) y Vikalpa (2012).

En cuanto a la rentabilidad, se confirma que entre mayor sea el ROA, mayor será la revelación de informaciones sobre capital estructural tecnológico, caso contrario al ROE que resultó que entre mayor sea esta variable, menor será la revelación de este tipo de informaciones. Las investigaciones revisadas presentan discrepancias en cuantos a los resultados sobre estas variables. Rodríguez (2004) encontró relación positiva entre la rentabilidad del activo y del patrimonio y la revelación de informaciones sobre intangibles. Por su parte Macagnan (2009) encontró relación negativa. Fontana y Macagnan (2013) no encontraron relación entre la rentabilidad del activo y la revelación de información sobre intangibles.

La hipótesis antigüedad fue confirmada con relación positiva, confirmando los resultados de Macagnan (2009). La hipótesis cotización en más de una bolsa fue confirmada con relación positiva. Esta hipótesis no fue contrastada en ninguna de las investigaciones revisadas. La hipótesis crecimiento de los activos fue confirmada con relación negativa. Confirmaron estos resultados los obtenidos por Rodríguez (2004).Seguidamente se presenta la revisión de literatura, formulación de hipótesis, metodología, análisis de resultados, conclusiones y bibliografía. 


\section{REVISIÓN DE LITERATURA}

Con el advenimiento de las redes y la era de la información, existe un creciente reconocimiento de que la información pudiera ser la única ventaja competitiva sostenible e inimitable. (Mata; Fuerst \& Barney, 1995; Shapiro \& Varian, 1999; Wade \& Hulland, 2004; Tallon, Ramírez e Short, 2013). A través de las ventajas ofrecidas por la tecnología, las empresas pueden atender a los clientes a un menor costo. (Rasmusen \& Young, 2012). La capacidad de una organización para innovar está estrechamente ligada a su capital intelectual, o su capacidad de utilizar sus recursos de conocimiento. (Subramaniam \& Youndt, 2005). En este nuevo escenario las organizaciones bancarias necesitan establecer mecanismos para seguir siendo competitivas. También requerirían proyectar al mercado el uso eficiente de estas ventajas competitivas. Esto se pudiera lograr a través de una mayor transparencia en cuanto a la divulgación de información representativa del capital intelectual, en particular del capital estructural tecnológico. Este tipo de intangibles comprenden todo lo relacionado a la mejora de inversión en tecnología, procesos y sistemas. (Kamaluddin \& Abdul Rahman, 2009). La transparencia informativa lo que busca es reducir el problema de la asimetría de la información entre agentes y propietarios.

En este sentido, la información es fundamental en los mercados financieros, sobre todo por el hecho de que si los inversionistas manejan información diferente a la que conocen los administradores respecto a las ganancias, se podría agravar el problema de la asimetría de la información. (Diamond \& Verrecchia, 1991; Kim \& Verrecchia, 1994; Bhattacharya, Desai e Venkataraman, 2013). Esto, debido al hecho de que aquellos mejor informados tomarían mejores decisiones, y en detrimento de los menos informados, quienes probablemente sufrirían pérdidas, lo que afectaría la eficiencia del mercado. (Macagnan, 2007).

Esta investigación parte del presupuesto de la existencia de asimetría de información en las relaciones entre propietarios e inversionistas. La evidencia empírica demuestra que la asimetría de la información tiene consecuencias no deseadas: valoración ineficiente de las acciones, elevado costo de capital y beneficios excesivos para quienes tienen acceso privilegiado a la información. (Lev, 2001, 2003; Macagnan, 2009).

Las divulgaciones voluntarias de informaciones se asocian con una disminución de la asimetría de la información y una reducción en el costo del capital. (SHROFF, et al., 2013). Estas divulgaciones pudieran ayudar a reducir los conflictos de intereses entre agentes y propietarios, que según la teoría de agencia surgen como consecuencia de la asimetría de información (Jensen \& Meckling, 1976). El uso de incentivos para crear la alineación entre los principales y agentes es un mecanismo primario propuesto por la teoría de agencia (Gómez, Bermejo e Franco, 2010; Cuevas, Gómez e Wiseman, 2012), considerándose un factor influyente en cuanto al buen gobierno corporativo. (Clegg, 2010; Lan \& Heracleous, 2010; Cuevas, Gómez e Wiseman, 2012; Raelin \& Bondy, 2013). La existencia de transparencia y buenas prácticas de gobierno corporativo pudieran disminuir los comportamientos oportunistas por quienes poseen la mayor cantidad de información. Según la teoría de la agencia, el propietario puede minimizar el oportunismo estableciendo un contrato adecuado para reducir la asimetría de información, que favorece al agente. (Eisenhardt, 1989; Dawson, Watson e Boudreau, 2010;). De esta manera se pudieran reducir los conflictos de intereses y los costes de agencia. La asimetría de información se traduce en altos costos de agencia (Johansson \& Malmstrom, 2012).

La teoría de la agencia parte del presupuesto de que cuanto mayor sea la divulgación de informaciones, menores serán los costes de agencia. Un importante mecanismo para reducir los costes de agencia es a través de la divulgación de información de forma voluntaria. De esta manera la dirección de la empresa reduce la asimetría de información con los accionistas. (Firth, 1984, Chow \& Wong-Boren, 
1987; Cooke, 1989, Christopher \& Hassan, 1995; Hossain et.al,. 1995; Watson et al., 2002; Nagar et al., 2003; Firer \& Williams, 2006; Macagnan, 2007).

En contrapunto a la teoría de agencia surge la teoría del coste del propietario. Esta teoríaemerge de los planteamientos de Verrecchia, (1983); Fishman y Hagerty, (1989); Darrough y Stoughton, (1990); Wagenhofer, (1990) y Macagnan (2007); que parten del principio de que las organizaciones limitarían la divulgación voluntaria de información en los mercados de capitales. Estas limitaciones se deben a los costes que se originan al preparar y divulgar la información, al igual que por los costes surgidos por mostrar sus ventajas competitivas. La literatura existente sobre el tema demuestra que el incentivo para proporcionar voluntariamente información por segmentos en el mercado es en realidad limitado, por la existencia de los costes de propiedad. (Prencipe, 2004).

No se encontraron en las investigaciones revisadas entre el período 1961 a 2013 ninguna investigación que analizara la revelación voluntaria de informaciones específicamente en el sector bancario.

\section{FORMULACIÓN DE HIPÓTESIS}

La hipótesis tamaño, medida a través de la variable total de activos, ha sido la más contrastada como elemento condicionante de la divulgación de información. Tal ha sido el caso de las investigaciones de: Cerf (1961), Singhvi y Desai (1971), Buzby (1975), Belkaoui y Kahl (1978), Salamon y Dhaliwal (1980), McNally, et al. (1982), Chow y Wong-Boren (1987), Cooke (1989, 1992), Craswell y Taylor (1992), García y Monterrey (1993), Malone, et al. (1993), Mckinnon y Dalimunthe (1993), Wallace, Naser y Mora, (1994), Hossain, Perera y Rahman (1995), Raffournier (1995), Wallace \& Naser (1995), Aitken, Hooper y Pickering (1997), Inchausti (1997), Patton y Zelenka (1997), Carnaghan (1999), Bujaki y McConomy (2002), Camfferman y Cooke (2002), Ferguson, Lam y Lee (2002), Watson, Sirves y Marston (2002), Arvidsson (2003), Bozzolan, Favotto y Ricceri (2003), Rodríguez (2004), Gómez, Iñiguez y Poveda (2005), Mendez-da-Silva y Magalhaes (2005), Bronson (2006), Méndez da Silva y Martelanc (2006), Hossain (2008), Hidalgo y Meca, (2009), Mendes-da-Silva, Ferraz, Famá y Maluf (2009), Mukherjee, et al. (2010), Vikalpa (2012), Fontana \& Macagnan (2013).. De estas investigaciones, Carnaghan (1999), Arvidsson (2003), Bozzolan et al (2003), Rodríguez (2004), Hidalgo y Meca, (2009) y Vikalpa (2012), encontraron que existe relación positiva entre el tamaño del activo y la revelación de informaciones sobre recursos intangibles. Por otra parte, la investigación de Fontana \& Macagnan (2013) concluyó con relación negativa entre el tamaño y la revelación de informaciones sobre recursos intangibles. El resto de las investigaciones analizaron la revelación de informaciones en general. De acuerdo a los presupuestos de la teoría de la agencia (Jensen \& Meckling, 1976), entre mayor sea el tamaño de una organización, mayor debería ser la divulgación de información. Esto con el fin de mitigar la posibilidad de conflictos de intereses entre los dueños y aquellos que administran su propiedad. Las empresas de mayor tamaño manejan cantidades de contratos superiores a las pequeñas, por lo que las primeras deberían revelar mayor cantidad de informaciones de acuerdo a los presupuestos de la teoría de la agencia. (Macagnan, 2007). Pudiera suponerse entonces, que las organizaciones más grandes contarían con los recursos necesarios para recolectar y procesar las informaciones. Las empresas grandes, adicional al hecho de contar con personal capacitado para procesar la información, estarían en capacidad de contar con sistemas de información más sofisticados que las pequeñas empresas. (Camfferman \& Cooke, 2002). Las empresas pequeñas no estarían entonces en capacidad de solventar los costes de revelar gran cantidad de informaciones. 
(Buzby 1975; Cooke, 1989, 1992). Sumado a esto estarían corriendo el riesgo de perder sus ventajas competitivas. (Macagnan, 2007). En base a estos planteamientos, se formula la siguiente hipótesis:

$H_{1}$. El tamaño de los bancos está positivamente relacionado con la divulgación de información representativa del capital estructural tecnológico.

La hipótesis rentabilidad, como elemento condicionante de divulgación de información representativa de recursos intangibles fue contrastada por las investigaciones de Rodríguez (2004), Macagnan (2005, 2007, 2009), García Meca, et al. (2005), Macagnan y Rodríguez (2005), Hidalgo y Meca (2009), Vikalpa (2012) y Fontana y Macagnan (2013).. Rodríguez (2004) encontró relación positiva entre la rentabilidad y la revelación de información sobre intangibles, Por su parte, Macagnan (2005. 2007, 2009) encontró relación negativa en sus investigaciones al contrastar la rentabilidad como elemento condicionante de la revelación de intangibles. Las investigaciones de Hidalgo y Meca (2009) y Fontana y Macagnan (2013) no encontraron relación entre estas variables en sus investigaciones. De acuerdo con los presupuestos de la teoría de la agencia (Jensen \& Meckling, 1976), cuando una empresa obtiene una elevada rentabilidad sería recomendable publicar mayor cantidad de informaciones. Esto con el fin de justificar la situación económica de la empresa ante sus accionistas. Por otra parte, la propia rentabilidad de la empresa habla por sí sola de la situación económica de la empresa, por lo que revelar este tipo de informaciones significaría incurrir en costes innecesarios, según la teoría del coste del propietario (Verrecchia, 1983, Macagnan, 2007). Adicional a ello la divulgación de información tiene efectos adversos en la posición competitiva (Rodríguez, 2004; Macagnan, 2007). Se plantea la siguiente hipótesis:

$\mathrm{H}_{2}$. La rentabilidad de los bancos está positivamente relacionada con la divulgación de información representativa del capital estructural tecnológico.

La antigüedad de la empresa como elemento condicionante de la revelación de informaciones sobre recursos intangibles fue contrastada en las investigaciones de Bukh, Nielsen, Gormsen, y Mouritsen (2004), y Macagnan $(2007,2009)$. Bukh et al. (2004) no encontraron relación entre la antigüedad y la revelación de informaciones sobre intangibles, mientras que Macagnan $(2007,2009)$ concluye que la edad de la empresa es un elemento condicionante de la revelación de informaciones. De acuerdo con la teoría de la agencia (Jensen \& Meckling, 1976), las empresas con una gran cantidad de años de existencia deberían revelar mayor cantidad de informaciones sobre sus recursos intangibles. Esto podría ser interpretado como una señal de que la empresa es sólida en términos de inversión. (Macagnan, 2007). Por su parte las empresas relativamente nuevas en el mercado pudieran requerir divulgar más informaciones. Esto debido al riesgo que podría representar para un inversionista con características conservadoras. (Macagnan, 2007). Desde la perspectiva de la teoría del coste del propietario (Verrecchia, 1983, Macagnan, 2007), la cantidad de años reflejaría la solidez de la empresa, por lo que no sería necesario en incurrir en costos de divulgación. Se presenta la siguiente hipótesis:

$\mathrm{H}_{3}$. La antigüedad de los bancos está negativamente relacionada con la divulgación de información representativa del capital estructural tecnológico.

Macagnan $(2007,2009)$ y Fontana $(2011)$ contrastaron en sus investigaciones la hipótesis precio de la acción dividido entre su valor contable, como elemento condicionante de la divulgación de capital 
estructural, confirmando la relación positiva entre estas variables. La asimetría de información entre las empresas de alta tecnología y crecimiento es mayor que en las empresas consolidadas (Rylander \& Jacobsen, 2000, Macagnan, 2007). La dirección de la empresa buscaría minimizar los posibles conflictos de intereses con los inversores. Para ello justificarían que la diferencia entre el valor contable y el valor de mercado de la empresa se encuentra en los recursos intangibles no contabilizados. En base a los presupuestos de la teoría de la agencia (Jensen \& Meckling, 1976), entre mayor sea el precio de la acción dividido entre el valor contable, mayor debería ser la revelación de información. Con esto se reduciría el problema de asimetría de información y los costes de agencia. Se formula la siguiente hipótesis:

$\mathrm{H}_{4}$. La diferencia entre el precio de la acción dividido entre el valor contable de los bancos está positivamente relacionada con la divulgación de información representativa del capital estructural tecnológico.

La hipótesis pago de dividendos, como elemento condicionante la divulgación de información representativa del estructural tecnológico, fue contrastada por Macagnan (2007, 2009), y Herrera (2013). Ninguna de estas investigaciones encontró relación entre el pago de dividendos y la revelación de información sobre capital estructural. De acuerdo a la teoría de la agencia (Jensen \& Meckling, 1976), a través de la divulgación de informaciones sobre el pago de dividendos, los inversionistas estarían en capacidad de estimar sus posibles ingresos en el futuro, y por tanto se reduciría el problema de la asimetría de la información. A medida que la empresa aumenta los pagos de sus dividendos, el mercado lo pudiera considerar un indicativo de que la empresa es confiable. Así disminuiría la necesidad de divulgar informaciones, ya que esto, de acuerdo a la teoría del coste del propietario (Verrecchia, 1983; Macagnan, 2007) significaría en incurrir en costes innecesarios. En base a lo anterior, se formula la siguiente hipótesis:

$H_{5}$. El pago de dividendos de los bancos está positivamente relacionado con la divulgación de información representativa del capital estructural tecnológico.

La hipótesis emisión de nuevas acciones, como elemento condicionante de la divulgación de información sobre capital estructural tecnológico, fue contrastada en la investigación de Herrera (2013). En concordancia con la teoría de la agencia (Jensen \& Meckling, 1976), la divulgación de informaciones sobre la emisión de nuevas acciones mostraría al mercado que puede tener confianza en la empresa. En sentido opuesto, divulgar informaciones respecto a la emisión de nuevas obligaciones, generaría costes adicionales para la organización, según la teoría del coste del propietario (Verrecchia, 1983; Macagnan, 2007). Se formula la siguiente hipótesis:

$\mathrm{H}_{6}$. La emisión de nuevas acciones por parte de los bancos está positivamente relacionada con la divulgación de información representativa del capital estructural tecnológico.

Fontana (2011), Fontana y Macagnan (2013) y Herrera (2013) testaron la hipótesis dividendos por acción, como elemento condicionante de la divulgación de información sobre recursos intangibles. Con la divulgación de informaciones respecto al pago de dividendos, los accionistas pueden tener una panorámica respecto a la administración de la empresa; en el sentido en que trabaja por los intereses 
de sus dueños. A través de la divulgación de este tipo de informaciones se reduce el problema de la asimetría de la información, de acuerdo con la teoría de la agencia. (Jensen \& Meckling, 1976). De acuerdo a los presupuestos de la teoría del coste del propietario (Verrecchia, 1983; Macagnan, 2007), el solo hecho de divulgar informaciones sobre el pago de dividendos provocaría costes innecesarios, ya que los pagos de dividendos en si mostrarían la capacidad e la empresa de retribuir a sus accionistas, por depositar su confianza en ella. Se formula la siguiente hipótesis:

$\mathrm{H}_{7}$. Los dividendos por acción de los bancos están positivamente relacionados con la divulgación de información representativa del capital estructural tecnológico.

La hipótesis cotización en más de una bolsa, no fue encontrada como elemento condicionante de la divulgación de información sobre recursos intangibles, por las investigaciones revisadas. De acuerdo con la teoría de la agencia, al cotizar en más de una bolsa una empresa debería de divulgar mayor cantidad de informaciones y así reducir los costes de agencia. (Jensen \& Meckling, 1976). Habría muchos intereses en la empresa por diferentes tipos de inversionistas y una mayor cantidad de contratos. (Macagnan, 2007). La teoría del coste del propietario por su parte, plantea que no es necesaria la divulgación de informaciones, por el hecho de que al ampliar sus mercados la empresa está mostrando su propio crecimiento y no valdría la pena incurrir en costes innecesarios. (Verrecchia, 1983; Macagnan, 2007). Se formula la siguiente hipótesis:

$\mathrm{H}_{8}$. La cotización en más de una bolsa por parte de los bancos está positivamente relacionada con la divulgación de información representativa del capital estructural tecnológico.

La hipótesis crecimiento como elemento condicionante de la revelación de informaciones sobre recursos intangibles fue contrastada por las investigaciones de Rodríguez (2004) y Fontana y Macagnan (2013). La teoría de la agencia (Jensen \& Meckling, 1976), parte del presupuesto de que las empresas con un mayor crecimiento deberían publicar más informaciones que las empresas pequeñas, porque cuentan con mayor cantidad de contratos. Esto para evitar el problema de la asimetría de la información y por el posible surgimiento de conflictos de intereses entre agentes y accionistas (Macagnan, 2007). Desde otra perspectiva, la teoría del coste del propietario (Verrecchia, 1983; Macagnan, 2007), plantea que el propio crecimiento de las empresas habla por sí solo de su evolución positiva, por lo que no sería necesario incurrir en costos de divulgación. En base a lo anterior, se formulan la siguiente hipótesis:

$\mathrm{H}_{9}$. El crecimiento de los bancos está positivamente relacionado con la divulgación de información representativa del capital estructural tecnológico.

La relación positiva de las variables explicativas es medida en base a que entre mayor sea el tamaño de las mismas, mayor será la divulgación de información representativa del capital estructural tecnológico. Por otra parte, la relación negativa significa que entre mayor sea el tamaño de la variable explicativa, menor será la divulgación de este tipo de información. 


\section{METODOLOGÍA DE LA INVESTIGACIÓN}

Se presenta a continuación el diseño metodológico de esta investigación.

Modelo empírico y descripción de variables

La fórmula 1 presenta el modelo empírico utilizado para medir la divulgación de información representativa del capital estructural tecnológico.

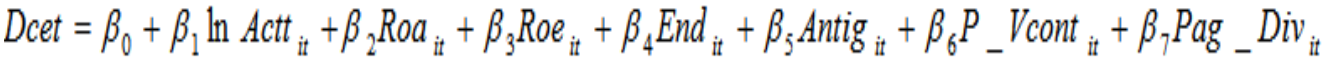

$$
\begin{aligned}
& +\beta_{8} E m_{-} N u_{-} A c c_{i t}+\beta_{9} D i v_{-} A c c_{i t}+\beta_{10} C o t_{-}+{ }_{-} B o l_{i t}+\beta_{11} C_{-} A c t t_{i t}+\beta_{12} C_{-} I n g_{i t}+\beta_{13} C L u_{i t}+\varepsilon_{i t}
\end{aligned}
$$

Siendo:

Dcet= divulgación de información representativa del capital estructural tecnológico

$\beta_{0}=$ constante

$B_{i}=$ parámetros que serán estimados

LnActt $_{\text {it }}=$ total del activo del banco "i" en el año " $\mathrm{t}$ ";

Roa $_{i t}=$ rentabilidad del activo del banco "i" en el año " $t$ ";

Roe $_{i t}=$ rentabilidad del patrimonio del banco "i" en el año " $\mathrm{t}$ ";

$\mathrm{End}_{\mathrm{it}}=$ endeudamiento del banco "i" en el año " $\mathrm{t}$ ";

Antig $_{\mathrm{it}}=$ antigüedad del banco "i" en el año " $\mathrm{t}$ ";

P_Vcont $t_{i t}=$ precio de la acción dividido entre su valor contable del banco "i" en el año " $t$ ";

Pag_Div ${ }_{i t}=$ dividendos pagados del banco "i" en el año " $t$ ";

$E m \_N u \_A c c$ it = emisión de nuevas acciones del banco "i" en el año "t";

Div_Acc ${ }_{\text {it }}=$ dividendos por acción del banco "i" en el año "t";

Cot_+_Bol $\mathrm{it}_{\mathrm{t}}=$ cotización en más de una bolsa del banco "i" en el año " $\mathrm{t}$ ";

C_Actt ${ }_{\text {it }}=$ crecimiento del activo total del banco "i" en el año " $\mathrm{t}$ ";

C_Ing ${ }_{\mathrm{it}}=$ crecimiento de los ingresos del banco "i" en el año "t";

$C_{-}$Luc $_{\text {it }}=$ crecimiento del lucro líquido del banco "i" en el año " $\mathrm{t}$ ";

$\varepsilon_{\text {it }}=$ error aleatorio que capta el efecto de las variables omitidas del banco "i" en el año "t";

$\mathrm{i}=$ bancos, de 1 a 29;

$\mathrm{t}=$ anos, de 2006 a 2011.

Variable dependiente: divulgación de información representativa del capital estructural tecnológico.

Para la medición de la divulgación de información representativa del capital estructural tecnológico, fueron creados veinte (20) indicadores, tal como se muestra en la tabla 1. Estos indicadores están basados en el modelo Macagnan (2007), quien utilizó 23 indicadores para analizar la revelación de capital estructural tecnológico de las empresas listadas en las bolsas de valores: de Barcelona y de Madrid. Para esta investigación se consideraron algunos indicadores del Modelo Macagnan, se incluyeron otros adicionales, adecuándoles al objetivo de este estudio, debido a que en esta investigación la muestra la integran las organizaciones bancarias. Estos indicadores representan valores tecnológicos 
de la empresa, e incluyen inversiones en investigación y desarrollo, licencias, patentes y la evolución del sistema productivo.

Tabla 1: Indicadores representativos del capital estructural tecnológico

Descripción y razones para las inversiones en tecnologías de información

Descripción de la(s) inversión(es) en hardware

Costos de la(s) inversión(es) en hardware

Descripción de la(s) inversión(es) en software

Costos de la(s) inversión(es) en software

Gastos de desarrollo/gastos de administración

Política, estrategia y/u objetivos de actividades de I+D

Fuerza competitiva de actividades de I+D respecto a los competidores

Perspectivas futuras con respecto a I+D

Gastos de inversión en I+D

Número de empleados en I+D

Número de licencias

Licencias pendientes

Detalle de licencias de la compañía

Nuevos productos/servicios

Productos/ servicios en desarrollo

Tasas de los servicios comercializados

Fase del ciclo de vida en que se encuentran los servicios

Características de los productos/ servicios ofertados

Indicadores de eficiencia de los productos/ servicios

Fuente: elaboración propia de las autoras

Para el desarrollo de este estudio, fue adoptado el procedimiento dicotómico, en el cual se utilizan uno o cero para dar a conocer la divulgación. Con el número uno se identifican los indicadores (ítems) revelados y con el cero los no revelados. (Cooke, 1989, 1992; Camfferman; Cooke, 2002; Macagnan, 2007, 2009) y se define como:

(2)

$$
\text { Ij }=\sum_{i=1}^{n j} \chi i j
$$

$n j$

Donde:

I= índice

$\mathrm{j}=$ banco

$\mathrm{i}=$ indicadores " $\mathrm{i}$ " del banco "j"

$\mathrm{xij}=$ número de indicadores "i" obtenidos para el banco " $\mathrm{j}$ "; 1 si el ítem es revelado, 0 si no es revelado; de modo que $\mathbf{O} \leq \boldsymbol{I} \leq 1$

$\mathrm{nj}=$ número de indicadores $=20$

Finalmente se divide el valor total de los ítems para cada banco entre el número máximo de los mismos que se puede lograr. Así se obtiene el porcentaje de divulgación para cada uno de los indicadores. 


\section{Variables explicativas}

Las variables explicativas están representadas por características de las organizaciones, que pudieran están influyendo en la divulgación de información representativa del capital estructural tecnológico. En la Tabla 2 se presentan las hipótesis, variables, identificación, fórmulas y unidades utilizadas.

Tabla 2: Hipótesis, variables, identificación, fórmulas y unidades utilizadas.

\begin{tabular}{|c|c|c|c|c|}
\hline Hipótesis & Variables & Identificación & Fórmulas & $\begin{array}{l}\text { Unidades } \\
\text { utilizadas }\end{array}$ \\
\hline Tamaño & Total de activos & Lnactt & Valor total del activo & $\begin{array}{c}\text { Millones de Re- } \\
\text { ales }\end{array}$ \\
\hline \multirow[t]{2}{*}{ Rentabilidad } & Roa & Roa & (Utilidad Neta/ Activos) $^{*} 100$ & Porcentual \\
\hline & Roe & Roe & $\begin{array}{c}\text { (Utilidad Neta/ Patrimonio Líqui- } \\
\text { do)* } 100\end{array}$ & Porcentual \\
\hline Antigüedad & $\begin{array}{c}\text { Años de constitución } \\
\text { del banco }\end{array}$ & Antig & Años de constitución del banco & Años \\
\hline $\begin{array}{c}\text { Precio de la acción dividido } \\
\text { por su valor contable }\end{array}$ & $\begin{array}{c}\text { Precio de la acción } \\
\text { dividido por su valor } \\
\text { contable }\end{array}$ & P_Vcont & $\begin{array}{c}\text { (Cotización de la acción /Valor con- } \\
\text { table de la acción)*100 }\end{array}$ & Porcentual \\
\hline Pago de dividendos & Pago de dividendos & Pag_Div & Pago de dividendos & Dicotómica \\
\hline Emisión de nuevas acciones & $\begin{array}{c}\text { Emisión de nuevas ac- } \\
\text { ciones }\end{array}$ & Em_Nu_Acc & Emisión de nuevas acciones & Dicotómica \\
\hline Dividendo por acción & Dividendo por acción & Div_Acc & Dividendo por acción & Reales \\
\hline $\begin{array}{c}\text { Cotización en más de una } \\
\text { bolsa }\end{array}$ & $\begin{array}{c}\text { Cotización en más de } \\
\text { una bolsa }\end{array}$ & Cot_+_Bol & Cotización en más de una bolsa & Dicotómica \\
\hline \multirow{3}{*}{ Crecimiento } & Activo total & C_Actt & $\begin{array}{c}\text { (Activo total del año analizado/acti- } \\
\text { vo total del año anterior) }{ }^{*} 100\end{array}$ & Porcentual \\
\hline & Ingresos & C_Ing & $\begin{array}{c}\text { (Ingresos del año analizado/ingresos } \\
\text { del año anterior)* }{ }^{*} 100\end{array}$ & 5 Porcentual \\
\hline & Lucro líquido & C_Luc & $\begin{array}{l}\text { (Lucro líquido del año analizado/ } \\
\text { lucro líquido del año anterior)*100 }\end{array}$ & Porcentual \\
\hline
\end{tabular}

Fuente: elaboración propia de las autoras

En el caso de esta investigación, se utilizó el real brasilero como moneda estándar para mostrar los datos, esto debido a que la mayor parte de la muestra está integrada por bancos de Brasil. Para ello, se utilizó el tipo de cambio promedio de cada año de las diferentes monedas, en relación con el real brasilero.

\subsection{Selección de la muestra}

Para el desarrollo de esta investigación, se han seleccionado los bancos inscritos en el mercado de capitales: en Brasil la bolsa de São Paulo - BM\&F BOVESPA y en España: las bolsas de Madrid y BarceIona. Fueron seleccionados los bancos de estos países debido a la proximidad en cuanto al volumen de negociación de ambos países. Se seleccionó el segmento bancario debido al hecho de que estas 
instituciones deben mostrar la mayor transparencia al mercado, ya que no sólo utilizan el dinero de los accionistas para financiarse, sino también el dinero de sus clientes. Adicional a ello, por los escándalos bancarios que se han producido en los últimos años. La muestra la integran aquellos bancos que publicaron el 100\% de sus informes anuales disponibles para descargar su página electrónica de internet, siendo 38 bancos. Fueron eliminados 9 de ellos, dada la ausencia de datos sobre algunas variables. Otros, porque los datos se encontraban muy dispersos de la media, lo cual pudiera ocasionar que se obtuvieran resultados poco significativos. La muestra quedó integrada por 29 bancos. Esta representa un total de 174 informes anuales. Esta muestra fue considerada para el análisis de las estadísticas descriptivas. Para el desarrollo de esta investigación, fueron analizados seis años, del 2006 hasta el 2011. El total de la muestra para cada uno de estos países se presenta en la Tabla 3.

Tabla 3: Representación de los bancos por país.

\begin{tabular}{|l|c|c|}
\hline \multicolumn{1}{|c|}{ País } & Total de bancos & Muestra \\
\hline Brasil & 29 & 18 \\
\hline España & 23 & 11 \\
\hline Total de bancos & 52 & 29 \\
\hline
\end{tabular}

Fuente: elaboración propia de las autoras

Para el análisis de regresión fueron eliminados dos bancos: uno de Brasil y uno de España, esto debido a que uno cotizaba en las bolsas de valores de ambos países y el otro era una subsidiaria, por lo cual se podrían comprometer los resultados. La muestra para la regresión quedó constituida finalmente por 27 bancos, representados por 162 informes anuales.

\section{RESULTADOS}

En esta sección se presentan los resultados obtenidos en esta investigación. Se muestran las estadísticas descriptivas y el análisis de correlación entre las variables explicativas, seguido del análisis de regresión lineal múltiple con datos de panel.

\subsection{Estadísticas descriptivas y análisis de correlación}

En este apartado se muestran las estadísticas descriptivas de las variables dependientes y explicativas. En la tabla 4 se muestran las estadísticas descriptivas de la divulgación de información representativa del capital estructural tecnológico de los bancos, en el mercado de capitales de BM\&F BOVESPA.

Tabla 4: Resumen de las estadísticas descriptivas de la divulgación de información representativa del capital estructural tecnológico de los bancos registrados en el mercado de capitales de Brasil. Bolsa de Valores de São Paulo - BM\&F BOVESPA.

\begin{tabular}{|c|c|c|c|c|c|}
\hline Año & N & Media & Desv-Std. & Mínimo & Máximo \\
\hline 2006 & 18 & 31.11 & 21.80 & 0.00 & 70.00 \\
\hline 2007 & 18 & 33.33 & 20.36 & 0.00 & 70.00 \\
\hline 2008 & 18 & 38.33 & 20.36 & 0.00 & 65.00 \\
\hline 2009 & 18 & 33.61 & 19.16 & 0.00 & 65.00 \\
\hline 2010 & 18 & 30.28 & 19.29 & 0.00 & 55.00 \\
\hline 2011 & 18 & 25.56 & 20.64 & 0.00 & 60.00 \\
\hline
\end{tabular}

Fuente: elaboración propia de las autoras en base a la información presentada en los informes anuales publicados por los bancos registrados en el mercado de capitales de BM\&F BOVESPA, el 2 de junio de 2012. 
De acuerdo a los resultados presentados en la tabla 4, se observa que los bancos de Brasil presentan un porcentaje de $0 \%$ como mínimo de revelación durante los cinco años de estudio. Esto genera como resultado una media de alrededor del 32\%, la cual es relativamente baja en cuanto a la revelación de información sobre capital estructural tecnológio por parte de los bancos brasileros. Dado que el máximo de revelación se encuentra entre el 55 y el 70\%, la desviación estándar es bastante significativa.

En la tabla 5 se muestran las estadísticas descriptivas de la divulgación de información representativa del capital estructural tecnológico los bancos en el mercado de capitales de Madrid y Barcelona.

Tabla 5: Resumen de las estadísticas descriptivas de la divulgación de información representativa del capital estructural tecnológico de los bancos registrados en el mercado de capitales. Bolsas de Madrid y Barcelona.

\begin{tabular}{|l|l|l|l|l|l|}
\hline \multicolumn{1}{|c|}{ Año } & \multicolumn{1}{c|}{$\mathrm{N}$} & \multicolumn{1}{c|}{ Media } & \multicolumn{1}{c|}{ Desv.Std. } & \multicolumn{1}{c|}{ Mínimo } & \multicolumn{1}{c|}{ Máximo } \\
\hline 2006 & 11 & 55.45 & 16.80 & 25.00 & 75.00 \\
\hline 2007 & 11 & 55.00 & 18.57 & 15.00 & 75.00 \\
\hline 2008 & 11 & 49.55 & 16.95 & 15.00 & 70.00 \\
\hline 2009 & 11 & 50.45 & 18.09 & 10.00 & 70.00 \\
\hline 2010 & 11 & 50.45 & 15.24 & 20.00 & 70.00 \\
\hline 2011 & 11 & 36.82 & 18.88 & 5.00 & 60.00 \\
\hline
\end{tabular}

Fuente: elaboración propia, en base a la información presentada en los informes anuales publicados por los bancos registrados en el mercado de capitales: Bolsas de Madrid y Barcelona, el 2 de junio de 2012.

De acuerdo a los resultados, la media de revelación de información sobre capital estructural tecnológico se mantiene alrededor del 50\%, excepto para el año 2011, que resultó con $36.82 \%$. En este año se muestra el menor porcentaje de la revelación con el $5 \%$. Al comparar con los resultados de Brasil, los bancos españoles en media revelan más informaciones sobre su capital estructural tecnológico. Adicionalmente, resultados muestran que existe un porcentaje de desviación estándar inferior al presentado en los resultados de los bancos brasileños. Esto por el hecho de que el porcentaje mínimo de revelación se aleja de cero (0), sin embargo el nivel máximo de revelación es muy próximo para ambos países.

La Tabla 6 muestra las estadísticas descriptivas de las variables explicativas.

Tabla 6: Estadísticas descriptivas de las variables explicativas.

\begin{tabular}{|l|c|c|c|c|c|}
\hline Variables Explicativas & $\mathrm{N}$ & Media & Desv-Std. & Mínimo & Máximo \\
\hline Actt & 161 & $287,000,000$ & $575,000,000$ & $1,120,649$ & $3,080,000,000$ \\
\hline Roa & 161 & 1.54 & 0.85 & -0.70 & 4.50 \\
\hline Roe & 161 & 15.49 & 7.65 & -5.10 & 41.40 \\
\hline Antig & 161 & 75.00 & 45.00 & 9.00 & 203.00 \\
\hline P_Vcont & 161 & 1.49 & 1.04 & 0.00 & 4.96 \\
\hline Div_Acc & 161 & 0.62 & 0.63 & 0.00 & 3.49 \\
\hline C_Actt & 161 & 21.50 & 40.27 & -55.25 & 430.39 \\
\hline C_Ing & 161 & 15.72 & 28.33 & -92.52 & 120.17 \\
\hline C_Luc & 161 & 20.89 & 76.57 & -209.31 & 616.98 \\
\hline
\end{tabular}

Fuente: elaboración propia de las autoras. 
Para el análisis de las estadísticas descriptivas de las variables explicativas, no fueron consideradas las variables dicotómicas. La variable total de activos muestra la existencia de una elevada desviación estándar en cuanto al tamaño de los bancos, encontrándose dentro de la muestra bancos muy grandes como muy pequeños. En cuanto a la eficacia para lograr utilidades a través de los activos, se observa una rentabilidad en promedio de $1.54 \%$, y algunos bancos de la muestra reflejan porcentajes negativos de rentabilidad. En cuanto a la rentabilidad del patrimonio, se observa una media aceptable, lo que indica un buen rendimiento de la inversión de los propietarios.

A través de la variable antigüedad, se observa que en la muestra existen bancos relativamente jóvenes, así como bancos con centenares de existencia. La relación precio de la acción/ valor contable muestra que en promedio las acciones están en un $0.49 \%$ por encima de su valor contable. En cuanto los dividendos pagados por acción, se observa una desviación estándar del $0.63 \%$, con pago máximo de 3.49 y un mínimo de 0 . En cuanto a las variables crecimiento del activo, de los ingresos y del lucro líquido, se observa que la mayor desviación estándar la muestra la variable crecimiento del lucro líquido, con el $76.57 \%$, mostrando así bancos con muy crecimiento de sus utilidades y otros con pérdidas significativas.

Para la variable total de activos, se sugiere la transformación de los datos originales en logaritmo o raíz cuadrada a fin de facilitar el trabajo estadístico. Para el resto de las variables explicativas se ajustó cada variable a menos uno, es decir ajustada al período anterior, a fin de lograr resultados favorables para el modelo de regresión. La Tabla 7 presenta los coeficientes de correlación entre las variables explicativas.

Tabla 7: Matriz de correlaciones de las variables explicativas.

Covariance Analysis: Ordinary

Sample (adjusted): 1161

Included observations: 155

after adjustments

\begin{tabular}{|c|c|c|c|c|c|c|c|c|c|c|c|c|}
\hline Correlation & $\underset{x}{E}$ & 홍 & 쏭 & $\sum_{x=x}^{O}$ & 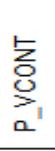 & 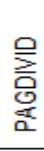 & 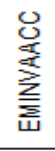 & 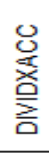 & 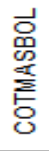 & 式 & 은 & $\begin{array}{l}\overrightarrow{\mathrm{J}} \\
\mathrm{J}\end{array}$ \\
\hline $\mathrm{ACTT}$ & 兽 & & & & & & & & & & & \\
\hline ROA & ণ্ণ & 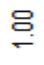 & & & & & & & & & & \\
\hline ROE & ర్ర & $\bar{s}$ & $\stackrel{8}{\circ}$ & & & & & & & & & \\
\hline ANTIG & 总 & 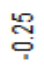 & $\frac{\infty}{6}$ & 음 & & & & & & & & \\
\hline P_VCONT & 응 & $\frac{2}{5}$ & 酸 & 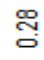 & $\stackrel{\circ}{\circ}$ & & & & & & & \\
\hline PAGDIVID & $\cong$ & ণ్రి & $\frac{\infty}{\circ}$ & $\approx$ & స్రి & $\stackrel{\circ}{\circ}$ & & & & & & \\
\hline EMINVAACC & ల్ల్ & 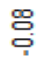 & $\bar{ড}$ & $\stackrel{\oplus}{\circ}$ & ले & $\stackrel{2}{\stackrel{0}{0}}$ & 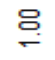 & & & & & \\
\hline DIVIDXACC & 哭 & $=$ & $\frac{\infty}{\circ}$ & ले & $\frac{6}{0}$ & 공 & $\frac{ \pm}{0}$ & $\stackrel{\circ}{\circ}$ & & & & \\
\hline COTMASBOL & 管 & ণָ & $\frac{20}{6}$ & 肙 & $\stackrel{\stackrel{్}{S}}{\circ}$ & $\stackrel{\check{O}}{\mathrm{O}}$ & 哭 & $\tilde{\Xi}$ & 음 & & & \\
\hline CACT & 号 & $\cong$ & $\cong$ & 응 & $\stackrel{2}{\stackrel{0}{0}}$ & ㅇㅇㅇ & $\frac{2}{0}$ & 용 & $\bar{O}$ & 음 & & \\
\hline CING & $\cong$ & 응 & 总 & $\frac{\infty}{0}$ & 号 & 동 & 잉 & 号 & ণ্ & ల్ల & 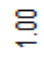 & \\
\hline CLUCL & 음 & $\frac{\mathscr{D}}{\circ}$ & $\approx$ & Бㅇㅁ & $\frac{0}{5}$ & ¿̊. & 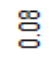 & 잉 & 음 & $\frac{6}{6}$ & ָே & $\stackrel{\circ}{\circ}$ \\
\hline
\end{tabular}


La variable activo total está relativamente correlacionada con la variable antigüedad. La variable Roa está relativamente correlacionada con la variable Roe. La variable Roe está relativamente relacionada con la variable precio entre el valor contable. Sin embargo estas correlaciones no son tan fuertes como para que exista la necesidad de eliminar alguna de las variables explicativas. En relación a las demás variables, se observan diferentes correlaciones en menor significancia.

\subsection{Análisis de regresión múltiple con datos de panel}

En esta sección se presentan los resultados del modelo de regresión con el métódo de mínimos cuadrados con datos de panel. La Tabla 8 muestra los resultados del modelo.

Tabla 8: Resultados del Modelo.

Dependent Variable: RCET

Method: Least Squares

Sample (adjusted): 2162

Included observations: 155 after adjustments

\begin{tabular}{|l|l|l|l|l|}
\hline \multicolumn{1}{|c|}{ Variable } & \multicolumn{1}{c|}{ Coefficient } & \multicolumn{1}{c|}{ Std. Error } & \multicolumn{1}{c|}{ t-Statistic } & \multicolumn{1}{c|}{ Prob. } \\
\hline LOG(ACTT) & 4.768011 & 0.838003 & 5.689728 & $* * * 0.0000$ \\
\hline ROA(-1) & 8.270418 & 2.052072 & 4.030277 & $* * * 0.0001$ \\
\hline ROE(-1) & -0.932283 & 0.259188 & -3.596932 & $* * * 0.0004$ \\
\hline ANTIG(-1) & 0.073043 & 0.035350 & 2.066250 & $* * 0.0406$ \\
\hline P_VCONT(-1) & 2.299486 & 1.677069 & 1.371134 & 0.1725 \\
\hline PAGDIVID(-1) & -2.665932 & 4.280411 & -0.622822 & 0.5344 \\
\hline EMINVAACC(-1) & 2.694105 & 3.194588 & 0.843334 & 0.4005 \\
\hline DIVIDXACC(-1) & -0.067006 & 2.272240 & -0.029489 & 0.9765 \\
\hline COTMASBOL(-1) & 7.700525 & 4.420724 & 1.741915 & $* 0.0837$ \\
\hline CACT(-1) & -0.114692 & 0.060358 & -1.900193 & $* 0.0594$ \\
\hline CING(-1) & 0.058708 & 0.049087 & 1.196020 & 0.2337 \\
\hline CLUCL(-1) & -0.013982 & 0.018359 & -0.761575 & 0.4476 \\
\hline C & -54.49995 & 15.38559 & -3.542272 & 0.0005 \\
\hline R-squared & 0.485355 & F-statistic & & 11.15986 \\
\hline Adjusted R-squared & 0.441864 & Prob(F-statistic) & & 0.000000 \\
\hline & & Durbin-Watson & & 2.215719 \\
\hline
\end{tabular}

${ }^{* * *} p<0.01,{ }^{* *} p<0.05,{ }^{*} p<0.10$

Fuente: elaboración propia de las autoras.

Este modelo no presenta problemas de autocorrelación, de acuerdo a los resultados del test de Correlación LM. De acuerdo con el estadístido Durbin Watson, los residuos son independientes.

Breusch-Godfrey Serial Correlation LM Test:

\begin{tabular}{|llll|}
\hline F-statistic & 0.843105 & Prob. $F(2,140)$ & 0.4325 \\
\hline Obs*R-squared & 1.844658 & Prob. Chi-Square(2) & 0.3976 \\
\hline
\end{tabular}


Las variables explican en un 44.19\% la varianza de la variable dependiente, de acuerdo al coeficiente de determinación R2. Se confirman las hipótesis tamaño, ROA, ROE, antigüedad, cotización en más de una bolsa y crecimiento del activo total como elementos condicionantes de la divulgación de información representativa del capital estructural tecnológico. Las hipótesis tamaño, ROA y ROE fueron confirmadas con un nivel de significancia de 1\% (p-value<0.01). La hipótesis antigüedad fue confirmada con el $5 \%$ de significancia ( $p$-value<0.05). Las hipótesis cotización en más de una bolsa y crecimiento del activo total fueron confirmadas un nivel de significancia de $10 \%$ (p-value<0.10). El resto de las hipótesis fueron rechazadas como elementos condicionantes de la divulgación de información representativa del capital estructural tecnológico.

La hipótesis tamaño, representada por el total de activos, fue confirmada como elemento condicionante de la divulgación de información representativa del capital estructural tecnológico, con relación positiva. Es decir, que entre mayor sea el tamaño de los bancos en Brasil y España, mayor será la revelación de este tipo de informaciones. Estos resultados corroboran los obtenidos por Cerf (1961), Singhvi y Desai (1971), Buzby (1975), Belkaoui y Kahl (1978), Salamon y Dhaliwal (1980), McNally, et al. (1982), Chow y Wong-Boren (1987), Cooke (1989, 1992), Craswell y Taylor (1992), Mckinnon y Dalimunthe (1993), Wallace et al. (1994), Hossain, et al. (1995), Raffournier (1995), Wallace y Naser (1995), Aitken et al. (1997), Inchausti (1997), Carnaghan (1999), Bujaki y McConomy (2002), Camfferman y Cooke (2002), Watson et al. (2002), Arvidsson (2003), Bozzolan et al. (2003), Rodríguez (2004), Gómez et al. (2005), Mendez-da-Silva y Magalhaes (2005), Barako et al. (2006), Bronson (2006), Méndez da Silva y Martelanc (2006), Hossain (2008), Hidalgo y Meca, (2009), Mendes-da-Silva et al. (2009), Vikalpa (2012).

La hipótesis rentabilidad del activo también fue confirmada para este modelo, con relación positiva. Significa esto que entre mayor sea el beneficio generado por los activos de los respectivos bancos, mayor sería la revelación de informaciones de recursos intangibles representativos de capital estructural tecnológico, recurso intangible no contabilizado. Esto confirma el presupuesto de la teoría de agencia, ya que los directivos de los bancos revelan informaciones voluntariamente sobre el capital estructural como forma de mostrar a los inversores que se encuentran operando según los intereses de los mismos. Las mismas conclusiones fueron obtenidas por Rodríguez (2004), Gómez et al. (2005), Vikalpa (2012).

La hipótesis Roe, representada por la variable rentabilidad del patrimonio, fue confirmada como elemento condicionante de la divulgación de información representativa del capital estructural tecnológico, con relación negativa. Es decir que entre mayor sea la capacidad de la empresa para remunerar a sus accionistas, menor sería la revelación de informaciones sobre capital estructural tecnológico, confirmando el presupuesto de la teoría del coste de propietario. Esto significa que los directivos de los bancos que presenten mayor rentabilidad del patrimonio consideran que el coste de revelación de informaciones sobre el capital estructural sería superior a los respectivos beneficios por revelar tales informaciones. Este resultado, ratifica los obtenidos por Belkaoui y Kahl (1978), Macagnan (2007) y Vikalpa (2012) en sus investigaciones.

Los resultados nos dejan la interrogante de porqué los directivos revelan más informaciones sobre su capital estructural tecnológico cuando la rentabilidad del activo es alta; sin embargo, cuando se obtiene una elevada rentabilidad del patrimonio se muestran menos informaciones sobre este tipo de recursos intangibles.

La hipótesis antigüedad fue confirmada con relación positiva. Esto significa, que los bancos más antigüos revelan mayor cantidad de informaciones sobre su capital estructural tecnológico que los bancos más jóvenes; ratificando los presupuestos de la teoría de la agencia. 
La hipótesis cotización en más de una bolsa, fue confirmada como elemento condicionante de la divulgación de información representativa del capital estructural tecnológico, con relación positiva. Es decir, que los directivos de los bancos investigados, que cotizan en más de una bolsa, revelarían voluntariamente más informaciones sobre su capital estructural tecnológico como presupone la teoría de agencia. Significa esto que los directivos reducirían la asimetría de información, enseñando que están operando según los intereses de accionistas o inversores.

La hipótesis crecimiento de los activos fue confirmada con relación negativa. Significa que entre mayor sea el crecimiento de los activos de los bancos de Brasil y España, menor será la revelación de sus informaciones representativas de capital estructural tecnológico. Estos resultados ratifican los presupuestos de la teoría del coste del propietario, siendo el crecimiento una buena señal de la situación financiera de la empresa, por lo que no se requiere incurrir en costes innecesarios de revelar este tipo de informaciones. Estos resultados van en sentido opuesto al hecho de que, los bancos españoñes y brasileros con mayor tamaño de sus activos con mayor rentabilidad de los mismos, revelan más informaciones sobre sus recursos intangibles relativos a capital estructural tecnológico.

Las hipótesis precio de la acción dividido entre su valor contable, pago de dividendos, emisión de nuevas acciones, dividendos por acción, crecimiento de los ingresos y crecimiento del lucro líquido fueron rechazadas como elementos condicionantes de la divulgación de información representativa del capital estructural tecnológico.

\section{CONSIDERACIONES FINALES}

La diferencia existente entre el valor contable y el valor de mercado de las empresas, es un problema que enfrenta la profesión contable. Por ello, la divulgación voluntaria de recursos intangibles ofrecería a los inversionistas una perspectiva de la situación real de la empresa, para que puedan tomar sus decisiones de inversión con la menor asimetría de información posible.

En base a los resultados de las estadísticas descriptivas, los bancos españoles reflejan un mayor porcentaje de la media en cuanto a divulgación de informaciones representativas del capital estructural tecnológico que los bancos brasileros. Así, los bancos en España han mostrado mayor preocupación por la transparencia informativa, en cuanto a divulgaciones voluntarias de informaciones representativas del capital estructural tecnológico.

Los resultados indican que entre mayor sea el tamaño de los bancos, más antigüos sean, mayor la rentabilidad del activo y en la medida en que coticen en más de una bolsa, mayor será la revelación de informaciones de recursos intangibles relativos a capital estructural tecnológico; ratificando así los presupuestos de la teoría de la agencia (Jensen \& Meckling, 1976). Los bancos con mayor rentabilidad de su patrimonio y con mayor crecimiento de sus activos, divulgan menos informaciones representativas del capital estructural tecnológico, confirmando los presupuestos de la teoría del coste del propietario (Verrecchia, 1983; Dye, 1986; Wagenhofer, 1990; Macagnan, 2007).

Las hipótesis precio de la acción dividido entre su valor contable, pago de dividendos, emisión de nuevas acciones, dividendos por acción, crecimiento de los ingresos y crecimiento del lucro líquido fueron rechazadas como elementos condicionantes de la divulgación de información representativa del capital estructural tecnológico.

Este estudio constituye un gran aporte al analizar mercados de capitales de dos países diferentes: Brasil y España. 


\subsection{Limitaciones de este estudio}

El informe anual es el único medio de revelación de información considerado en esta investigación; sin embargo las organizaciones bancarias que integran el mercado de capitales de estos países podrían utilizar otros medios para comunicar este tipo de informaciones a los interesados.

En este estudio sólo se analiza la revelación voluntaria de recursos intangibles relativos a capital estructural tecnológico. Sería importante hacer una comparación con otros índices de revelación, que podrían permitir conocer la importancia que las organizaciones bancarias dan a otros indicadores de recursos intangibles.

Este estudio se analiza solamente la revelación de información voluntaria, dejando de lado la información obligatoria. La comparación entre información obligatoria y voluntaria permitiría mayor claridad de la situación de los bancos en el mercado.

Comparar la información obligatoria que deben presentar los diferentes bancos en cada uno de los países,

Este estudio solamente consideró la información presentada, no así si la misma era completa o no.

No se trata de un abordaje comparativo entre estos dos países, sólo se analiza la correlación entre la divulgación de información de capital estructural tecnológico y los elementos que pudieran condicionar la misma. 


\section{REFERÊNCIAS}

Aitken, M., Hooper, C. e Pickering J. (1997). Determinants of voluntary disclosure of segment information: A re-examination of the role of diversification strategy. Accounting and Finance, 37(1), 89-109, May..

Akerlof, G. A. (1970). The market for lemons: quality uncertainty and the market mechanism. Quarterly Journal of Economics, 84(3), 488-500, Aug.

Arvidsson, S. (2003). The extent of disclosure on intangibles in annual reports. Working paper, 4th annual SNEE congress in Mölle.

Barako, D.G., Hancock P. \& Izan, H.Y. (2006). Factors influencing voluntary corporate disclosure by Kenyan companies. Corporate Governance: An International Review, 14(2), 107-125, doi: 10.1111/

j.1467-8683.2006.00491.x.

Belkaoui, A. \& Kahl, A. (1978). Corporate financial disclosure in Canada Research Monograph,

1. Canadian Certified General Accounting Association, Vancouver.

Belkaoui, A. \& Kahl, A. (1978). Corporate financial disclosure in Canada Research Monograph, 1. Canadian Certified General Accounting Association, Vancouver.

Bhattacharya, N., Desai, H. e Venkataraman, K. (2013). Does earnings quality affect information asymmetry? Evidence from Trading Costs. Contemporary Accounting Research, 30(2) 482-516, Summer. Disponível em: doi:10.1111/j.1911-3846.2012.01161.x.

Botosan, C. (1997). Disclosure level and the cost of equity capital. The Accounting Review, 72(3), 323-349, Jul.

Bozzolan, S., Favotto, F. e Ricceri, F. (2003). Italian annual intellectual capital disclosure: an empirical analysis. Journal of Intellectual Capital, 4(4), 543-558.

Brammer, S. \& Pavelin, S. (2006). Voluntary environmental disclosures by large UK companies. Journal of Business Finance \& Accounting, 33(7-8), 1168-1188, Sept.-Oct. Disponível em: http:// dx.doi.org/10.1111/j.1468-5957.2006.00598.x.

Bronson, S.N., Carcello, J.V. e Raghunandan, K. (2006). Firm characteristics and voluntary management reports on internal control. Auditing: A Journal of Practice \& Theory, 25(2), 25-39, Nov.

Bujaki, M. \& Mcconomy, B. J. (2002). Corporate Governance: Factors influencing voluntary disclosure by publicly traded Canadian firms. Canadian Accounting Perspectives, 1(2), 105-139.

Belkaoui, A. \& Kahl, A. (1978). Corporate financial disclosure in Canada Research Monograph,

1. Canadian Certified General Accounting Association, Vancouver.

Buzby, S.L. (1975). Company size, listed versus unlisted stock, and the extent of financial disclosure. Journal of Accounting Research, 13(1), 16-37, Spring.

Camfferman, K. \& Cooke, T.E. (2002). An analysis of disclosure in the annual reports of U.K. and Dutch companies. Journal of International Accounting Research, 1, 3-30. 
Carnaghan. (1999). Factors influencing managerial decisions about intangible asset disclosures: The role of accountability theory and impression management. University of Alberta.

Cerf, A. R. (1961). Corporate reporting and investment decisions, Berkely, California: The University of California Press. United States of America (USA).

Champlin, D. \& Knoedler, J. (Jun., 2003). Corporations, Workers, and the Public Interest. Journal of Economic Issues, 37(2), 305-313.

Chow, C. \& Wong-Boren, A. (1987). Voluntary financial disclosure by Mexican corporations. The Accounting Review, 62(3), 533-541, July.

Christopher T. \& Hassan, S. (1995). Voluntary disclosure of cash for information: Australian evidence. Working Paper. BAA Conference, Bristol.

Clegg, S. (2010). The state, power, and agency. Journal of Management Inquiry, 19, 4-13.

Cooke, T. E. (1989). Voluntary corporate disclosure by Swedish companies. Journal of International Financial Management \& Accounting, 1(2), 171-195, Summer.

(1992). The impact of size, stock market listing and industry type on disclosure in the annual reports of Japanese listed corporations. Accounting \& Business Research, 22(87), 229-237, Summer.

Craswell, A. T. \& Taylor, S. L. (1992). Discretionary disclosure of reserves by oil and gas companies: an economic analysis. Journal of Business Finance \& Accounting, 19(2), 295-308, Jan. Disponível em: doi: 10.1111/j.1468-5957.1992.tb00626.x.

Darrough, M. \& Stoughton, N. (1990). Financial disclosure policy in an entry game. Journal of Accounting \& Economics, 12(1-3), 219-243, Jan.

Dawson, G., Watson, R. e Boudreau, M. (2010). Information Asymmetry in Information Systems Consulting: Toward a Theory of Relationship Constraints. Journal of Management Information Systems, 27(3), 143-177, Winter. Disponível em: doi 10.2753/MIS0742-1222270306

Diamond, D. \& Verrecchia, R. (1991). Disclosure, liquidity and the cost of capital. The Journal of Finance, 46(4), 1325-1359, Sept.

Dye, R. A. Proprietary and nonproprietary disclosures. Journal of Business, v. 59, n. 2, p. 331-366, Apr. 1986.

Eisenhardt, K. M. (1989). Agency theory: an assessment and review. Academy of Management Review, 14(1), 57-74, Jan. Disponível em: doi: 10.5465/AMR.1989.4279003.

Ferguson, M. J., Lam, K. e Lee, G. M. (2002). Voluntary Disclosure by State-owned Enterprises Listed on the Stock Exchange of Hong Kong. Journal of International Financial Management and Accounting, 13(2), 125-152, Summer.

Firer, S. \& Williams, M. (2006). Association between ownership structures of Singapore publicy traded firms and intellectual capital disclosure. Corporate Governance and Intellectual Capital, 1-31, Archive. 
Firth, M. (1984). The extent of voluntary disclosure in corporate annual reports and its association with security risk measures. Applied Economics, 16(2), 269-277, Apr.

Fishman, Michael; Hagerty, L. (1989). Disclosure decisions by firms and the competition for price efficiency. The Journal of Finance, 44(3), 633-646, Jul.

Fontana, F. \& Macagnan, C. B. (2013). Factors explaining the level of voluntary human capital disclosure in the Brazilian capital market. Intangible Capital, 9(1), 305-321. Disponível em: http://dx.doi.org/10.3926/ ic.315.

Fontana, F. B. (2011). Fatores explicativos do nível de evidenciação voluntária de informações sobre recursos intangíveis das empresas listadas na BM\&F BOVESPA. Dissertação (Mestrado Programa de Pós-Graduação em Ciências Contábeis) - Universidade do Vale do Rio dos Sinos, Brasil.

García-Benau, M. A. \& Monterrey, J. (1993). Revelación voluntaria en las compañías españolas cotizadas en Bolsa. Revista Española de Financiación y Contabilidad, 23(74), 53-70, Jan-Mar.

Gómez, J., Iñiguez, R. e Poveda, F. (2005). Revelación voluntaria de información y características de las sociedades cotizadas en el mercado de capital español. Revista Española de Financiación y Contabilidad, 131, 8-32.

Herrera R., E. E. (2013). Revelación voluntaria de los recursos intangibles de los bancos: Panamá, Brasil y España y sus factores explicativos. Tesis doctoral. Universidad de Panamá, Panamá..

Hidalgo G., R. \& García, M. E. (2009). Divulgación de Información sobre El Capital Intelectual de Empresas Nacionales que Cotizan en la Bolsa Mexicana de Valores. Revista Contaduría y Administración, 229, 105-131, Sept.-Dec.

Hossain, M. (2008). The extent of disclosure in annual reports of banking companies: The case of India. European Journal of Scientific Research, 23(4), 659-680.

Hossain, M., Perera, M. H. B. e Rahman, A. R. (1995). Voluntary disclosure in the annual reports of voluntary disclosure in the annual reports of New Zealand companies. Joumal of Intemational Financial Management and Accounting, 6(1), 69-87, Spring.

Ichausti, B.G. (1997). The influence of company characteristics and accounting regulation on information disclosed by Spanish firms. The European Accounting Review, 6(1), 45-68. Disponível em: doi: 10.1080/096381897336863.

Jensen, M. C. \& Meckling, W. H. (1976). Theory of the firm: managerial behavior, agency costs and ownership structure. Journal of Financial Economics, 3(4), 305-360, Oct. 
Johansson, J. M. \& Malmstrom, M. (2012). The business model transparency paradox in innovative growth ventures: trade-offs between competitive advantages and agency costs. The Business Model Transparency Paradox, 3(2), p 238-263, Apr. Disponível em: doi 10.1515/erj-2012-0011.

Kamaluddin, A. \& Abdul Rahman, R. (2009). Enhancing organization effectiveness through human, relational and structural capital. An empirical analysis. Malaysian Accounting Review, 8(1), 1-17.

Kim, O. \& R. E. Verrecchia. (1994). Market liquidity and volume around earnings announcements. Journal of Accounting and Economics, 17(1), 41-67, Jan.

Klimontowicz, M. \& Harasim, J. (2013). The Identification of polish banks intangibles' significance and efficiency. Proceedings of the International. In: Conference on Intellectual Capital, Knowledge Management \& Organizational Learning, 231-240.

Klimontowicz, M. (2010). How intangibles affect the polish consumers' decisions on the banking market? Proceedings of the European Conference on Intellectual Capital, 337-345.

Lambert, R., Leuz, C. e Verrecchia, R. (2007). Accounting information, disclosure, and the cost of capital. Journal of Accounting Research, 45(2), 385-420, May. 2007. Disponível em: http://dx.doi.org/10.1111/ j.1475-679X.2007.00238.x

Lev, B. (2001). Intangibles: medición, gestión e información. Barcelona: Deusto.

. (2003). Intangible Assets: concepts and measurements. Enciclopedia of Social Measurement, 2.

Macagnan, C.B. (2005). Factores explicativos de la revelación de información de activos intangibles de empresas que cotizan en España. Trabajo de investigación - Universidad Autónoma de Barcelona, España.

. (2007). Condicionantes e implicación de revelar activos intangibles. Tesis doctoral. (Departamento de Economía de la Empresa) Universidad Autónoma de Barcelona, España.

(2009). Evidenciação voluntária: fatores explicativos da extensão da informação sobre recursos intangíveis. Revista Contabilidade \& Finanças, USP, São Paulo, 20(50), 46-61.

Malone, D., Fries, C. e Jones, T. (1993). An empirical investigation of the extent of corporate financial disclosure in the oil and gas industry. Journal of Accounting, Auditing and Finance, 8(3), 249-275, Summer.

Mata, F. J., Fuerst, W. L. e Barney, J. B. (1995). Information technology and sustained competitive advantage: A resource-based analysis. MIS Quarterly, 19(4), 415-433, Dec.

Mckinnon, H.; Dalimunthe, L. (1993). Voluntary disclosure of segment information by Australian diversified companies. Accounting and Finance, 33(1), 33-50, May. 
Mcnally, G. M., Eng, L. H. e Hasseldine, C. R. (1982). Corporate financial reporting in New Zealand: an analysis of user preferences, corporate characteristics and disclosure practices for discretionary information. Accounting and Business Research, 13(49), 11-20, Winter.

Mendes-da-Silva W. et al (2009). Disclosure via website corporativo: um exame de informações financeiras e de governança no mercado brasileiro. RAE-eletrônica, São Paulo, 49(2), 190-205, Apr.

Mendez Da Silva, W. \& Martelanc, R. (2006). Relacionamento com investidores com uso do website corporativo: análise empírica das empresas brasileiras listadas na Bovespa. Reflexão Contábil, 25(3), 41-56, Sept.-Dec.

Mendez-da-Silva; Magalhaes. (2005). Determinantes da disseminação voluntária de informações financeiras na internet. RAE-eletrônica, São Paulo, 4(2), Jul.-Dec.

Mukherjee, K., Sen, M. e Pattanayak, J. K. (2010). Firm characteristics and corporate environmental disclosure practices in India. Journal of Accounting Research \& Audit Practices, 9(4), 24-41, Oct.

Nagar, et al. (2003). Discretionary disclosure and stock-based incentives, Journal of Accounting and Economics, 34, 283-309.

Patton, J. \& Zelenka, I. (1997). An empirical analysis of the determinants of the extent of disclosure in annual reports of joint stock companies in the Czech Republic. The European Accounting Review, 6(4), 605-626, Sept.

Prencipe, A. (2004). Proprietary costs and determinants of voluntary segment disclosure: evidence from Italian listed companies. The European Accounting Review, 13(2), 319-340, Jul. Disponível em: doi: 10.10800963818042000204742.

Raffournier, B. (1995). The determinants of voluntary financial disclosure by Swiss listed companies. The European Accounting Review, 4(2), 261-280, Jul.

Rasmusen, E. \& Young, Y. (2012). First versus second mover advantage with information asymmetry about the profitability of new markets. The Journal of Industrial Economics, 60(3), 374-405, Sept.

Rodríguez, G. (2004). Factores explicativos de la revelación voluntaria de información sobre fuentes de ventaja competitiva empresarial. Revista Española de Financiación y Contabilidad, 33(122), 705-739, Jul-Sept.

Rowell, D. \& Connelly, L. B. (2012). A history of the term 'moral hazard'. Journal of Risk \& Insurance, 79(4), 1051-1075, Dec. Disponível em: doi:10.1111/j.1539-6975.2011.01448.x

Rylander, A. \& Jacobsen, K. (2000). Towards improved information disclosure on intellectual capital. International Journal of Technology Management, 20(5-8), 715-742. 
Salamon, G.L. \& Dhaliwal, D.S. (1980). Company size and financial disclosure requirements with evidence from the segmental reporting issue. Journal of Business Finance \& Accounting, 7(4), 555-568, Winter.

Seog, S. (2012). Moral hazard and health insurance when treatment is preventive. Journal of Risk \& Insurance, 79(4), 1017-1038, Dec. Disponível em: doi:10.1111/j.1539-6975.2011.01459.x

Shapiro, C. \& Varian, H. R. (1999). Information Rules: A Strategic Guide to the Network Economy. Boston: Harvard Business School Press.

Shroff, N. et al. (2013). Voluntary disclosure and information asymmetry. Evidence from the 2005 securities offering reform. Journal of Accounting Research, 51(5), 1299-1345, Dec. Disponível em: doi.10.1111/1475-679X.12022.

Singhvi, S. \& Desay, H. (1971). An empirical analysis of the quality of corporate financial disclosure. The Accounting Review, 46(1), 129-138, Jan.

Subramaniam, M. \& Youndt, M. (2005). The influence of intellectual capital on the types of innovative capabilities. Academy of Management Journal, 48(3), 450-463, Jun.

Tallon, P., Ramirez, R. e Short, J. (2013). The information artifact in it governance: toward a theory of information governance. Journal of Management Information Systems, 30(3), 141-177, Winter.

Verrecchia, R. E. (1983). Discretionary disclosure. Journal of Accounting \& Economics, 5(3), 179-194, Dec.

Vikalpa, R. (2012). Corporate disclosure of intangibles: a comparative study of practices among Indian, US, and Japanese companies. The Journal for Decision Makers, 37(3), 51-72, Jul.-Sept.

Wade, W. \& Hulland, J. (2004). The resource-based view and information systems research: Review, extension, and suggestions for future research. MIS Quarterly, 28(1), 107-142, Mar.

Wagenhofer, A. (1990). Voluntary disclosure with a strategic opponent. Journal of Accounting \& Economics, 12(4), 341-363, Mar.

Wallace, R. S. O. \& Naser, K. (1995) Firm- specific determinants of the comprehensiveness of mandatory disclosure in the corporate annual reports of firms listed on the Stock Exchange of Hong Kong. Journal of Accounting \& Public Policy, 14(4), 311-368, Winter.

Wallace, O., Naser, L. \& Mora, A. (1994). The relationship between the comprehensiveness of corporate annual reports and firm characteristics in Spain. Accounting and Business Research, 25(97), 41-53, Winter. Watson, A., Sirves, P. e Marston, C. (2002). Voluntary disclosure of accounting ratios in the UK. British Accounting Review, 34(4), 289-313, Dec. Disponível em: doi: 10.1006/bare.2002.0213.

Williams, S. M. (2001). Is intellectual capital performance and disclosure practices related? Journal of Intellectual Capital, 2(3), 192-203. 